\title{
eHealth innovations in LMICs of Africa and Asia: a literature review exploring factors affecting implementation, scale-up, and sustainability
}

This article was published in the following Dove Press journal:

Innovation and Entrepreneurship in Health

30 October 2015

Number of times this article has been viewed

\author{
Tanzir Ahmed Shuvo' \\ Rubana Islam' \\ Shahed Hossain' \\ Julie L Evans ${ }^{1,2}$ \\ Fatema Khatun ${ }^{1,3}$ \\ Tanvir Ahmed ${ }^{1,4}$ \\ Rukhsana Gazi' \\ Alayne MAdams' \\ 'Centre for Equity and Health \\ Systems, International Centre \\ for Diarrhoeal Disease Research, \\ Bangladesh (ICDDR,B), Mohakhali, \\ Dhaka, Bangladesh; ${ }^{2}$ Department \\ of Health Services Research and \\ Policy, London School of Hygiene \\ and Tropical Medicine, London, \\ UK; ${ }^{3}$ School of Public Health and \\ Community Medicine, The University \\ of New South Wales, Kensington, \\ NSW, Australia; ${ }^{4}$ Institute of \\ Development Studies, University of \\ Sussex, Brighton, East Sussex, UK
}

Correspondance:Alayne MAdams Centre for Equity and Health Systems, International Centre for Diarrhoeal Disease Research, Bangladesh (ICDDR,B), 68 Shaheed Tajuddin Ahmed Sarani, Mohakhali, Dhaka- 12 I2, Bangladesh Email aadams@icddrb.org
Background: In many low- and middle-income countries (LMICs), health system challenges relating to weak governance, health workforce shortages, and geographic and economic barriers to care impede effective delivery of health services to those in need. The rapid development of information and communication technologies over the last few decades offers the potential for addressing some of these challenges with innovative solutions, especially if offered at scale. This review reflects on the features of larger and more established eHealth interventions that may contribute to their utilization, scale-up and sustainability and, ultimately, to improved health outcomes.

Methods: Eight researchers conducted a literature review of eHealth innovations in LMICs of Asia and Africa. Peer-reviewed literature published between March 2010 and March 2015 was considered for inclusion in the review. Major online databases searched included Medline (via PubMed) and Web of Science. Some minor databases were also accessed. Articles addressing eHealth innovations were selected based on the following criteria: interventions located in LMICs of Asia and Africa; interventions of more than 1 year in duration; and interventions that cover at least one district or province of a country. Selected articles were analyzed and compared using a framework approach.

Results: Based on specified inclusion and exclusion criteria, 14 peer-reviewed articles (eight intervention studies, six reviews) were identified that reported on eHealth innovations. Six key dimensions were identified as influential for the successful implementation, utilization and scale-up of an eHealth innovation. eHealth projects need to: be designed in response to identified health needs and priorities; be supported by an enabling environment; ensure IT systems integration; establish effective partnership between stakeholders; ensure implementation requirements are met; and pre-empt and address issues related to end users' abilities to access, trust, accept, and utilize an eHealth product.

Conclusion: Consideration of the features identified in this review may be useful to health policy makers, program implementers, and innovators involved in the planning, design, and implementation of effective eHealth innovations intended to address large-scale population health needs in resource-constrained settings.

Keywords: eHealth, mHealth, telemedicine, HMIS, innovation, LMICs, developing countries

\section{Introduction}

Despite continuous efforts toward health improvement in low- and middle-income countries (LMICs), progress has been hindered by weak or dysfunctional health systems that fail to deliver quality, affordable health care to populations in need. Global health workforce shortages; geopolitical, economic, and environmental crises; geographic barriers; and weak governance are among the factors that are challenging effective health care delivery. ${ }^{1}$ 
One promising area of innovation is the application of information and communication technologies (ICTs) as a means to addressing some of these challenges, especially if such tools can be offered at scale. ${ }^{2}$ The potential of what ICTs can deliver to make health care more accessible, affordable and effective has led to a proliferation of new ideas and innovations, some of which are being implemented in LMICs. However, the small scale and pilot nature of many of these ICT interventions, colloquially referred to as "pilotitis", ${ }^{3}$ is widely considered a barrier to achieving sustainability at scale. ${ }^{4}$ A critical examination of some large-scale interventions might therefore contribute to identifying the features of ICT health interventions that can facilitate utilization, scale-up and sustainability.

Applications of ICT in the health sector are referred to as eHealth, defined by World Health Organization (WHO) as "the transfer of health resources and health care by electronic means". eHealth interventions encompass: 1) delivering health information for health professionals and health consumers through the Internet and telecommunication; 2) using IT and e-commerce to improve public health services, eg, through the education and training of health workers; and 3) applying e-commerce and e-business practices in health systems management. ${ }^{5}$ A subcomponent of eHealth is mHealth, which refers to "medical and public health practice supported by mobile devices, such as mobile phones, patient monitoring devices, personal digital assistants (PDAs), and other wireless devices" ${ }^{6}$ LMICs account for three-quarters of the rapidly expanding internet and mobile cellular subscriptions globally, ${ }^{7}$ thus creating opportunities for innovative and cost-effective health services through the use of ICT. They also provide scope for bringing health innovations closer to hard-to-reach populations who have restricted access to health care facilities. ${ }^{8,9}$

Some instances of eHealth applications in LMICs include increasing access to primary health care, providing realtime diagnosis and treatment, conveying health education, assisting in emergency medical response, and enabling data collection as part of disease surveillance. ${ }^{10,11}$

While eHealth is highlighted as an exciting area of innovation with potential for improving health outcomes, experience suggests that it is not a stand-alone solution for ailing health systems. ${ }^{10}$ Recent reviews on eHealth have explored some of the challenges in systems integration ${ }^{12,13}$ and other organizational barriers to health information technology uptake. ${ }^{14}$ Experts in the field also acknowledge the relative absence of rigorous evaluation research of such technologies on health outcomes. ${ }^{10}$
Existing evidence on eHealth interventions mostly focus on smaller-scale initiatives and their impacts on defined target populations. While there are relatively fewer number of promising larger-scale eHealth efforts, it is nevertheless interesting to consider the factors that have made them successful.

Recognizing the potential that these technologies have to offer when conceived as part of a systems approach to health care improvement, this literature review offers a synthesis and critical reflection about the features of larger and more established eHealth interventions that may contribute to utilization, scale-up, and sustainability and ultimately to improved health outcomes. While these observations are largely qualitative in nature, they may be useful to program planners and policy makers involved in designing eHealth strategies to strengthen health systems and support the goal of universal health care (UHC) in LMICs in Africa and Asia.

In this review, we will use the word eHealth to demonstrate all types of ICT-based health interventions including the mHealth approaches.

\section{Methods}

This narrative review identifies and synthesizes published studies that focus on larger and more established eHealth innovations in LMICs in Asia and Africa, as well as several reviews of eHealth interventions. In this review, we will interchangeably use the words "intervention" and "innovation" as the application of ICT in health is relatively new. Since this synthesis did not involve any human subjects, no ethical clearance was required.

\section{Search process}

The search was conducted in several steps. First, keywords and index terms for developing a comprehensive search strategy were identified. This was accomplished through a preliminary search of Medline and review of a sample of 179 abstracts from this search. This was followed by a consensus meeting among the eight-member review team, during which keywords, study objectives and inclusion and exclusion criteria were discussed and agreed.

The next step involved the development of a search strategy for Medline/PubMed. Index terms combined with selected Medical Subject Headings (MeSH) and free text terms related to the themes of telemedicine, eHealth, mHealth and ICT for health were applied using Boolean operators and wildcard truncation, as appropriate. Keywords were identified using the Population, Intervention, Comparison, and Outcomes (PICO) approach. The same PubMed search strategy was applied and modified, 
as required, for other major databases. Details on the PubMed search strategy used for this review are found in Web Annex 1.

Major databases searched were Medline/PubMed, Web of Science (including Science Citation Index Expanded) Social Science Citation Index (SSCI), CINAHL and VHL (including Cochrane Library, CENTRAL, CDSR-Cochrane Database of systematic reviews, and LILACS). For minor search engines with limited scope for advanced searching, such as WHOLIS/World Bank (Open Knowledge Repository), we utilized appropriate keywords applied one at a time or in combination.

\section{Study identification}

All citations obtained from the search were downloaded to Endnote X7 library to facilitate systematic screening. Although the eHealth field was spawned in the 1990s, support for its potential only gained momentum in the early $2000 \mathrm{~s}^{15,16}$ In 2005, the WHO passed World Health Assembly resolution WHA 58.28 which provided direction to member states on the use of eHealth. ${ }^{17}$ Thereafter, eHealth initiatives progressed rapidly in developed countries, and began to penetrate LMICs in Asia and Africa in the following decade. Considering this, we limited our study to the last 5 years from 2010 to 2015, when most eHealth development and implementation was occurring in these countries. We also limited our search to English language publications due to resource limitations and the language skills of the researchers involved. Studies were included if they met the following inclusion criteria:

1. Reported on eHealth innovations in LMICs in Asia and Africa (Annex 1 for search strategy).

2. Discussed issues related to intervention acceptability/ utilization/coverage/sustainability.

3. Reported on interventions operating for more than 1 year.

4. Reported on interventions applied in at least one district or province.

Given our particular interest in larger, more established eHealth interventions, criteria 3 and 4 served to remove smaller, short-term projects. Studies that did not meet these four inclusion requirements were excluded from consideration. In addition, we also considered a number of recent review papers discussing issues related to the acceptability, utilization, coverage and sustainability of eHealth interventions.

\section{Data abstraction and analysis}

Two reviewers initially screened paper titles and abstracts independently, then came together to make and justify the final selection. In cases of disagreement or confusion, a third reviewer was involved. Selected articles were analyzed and compared using a framework approach. This method involved summarizing and comparing case-based data through the use of data displays and summaries. ${ }^{18}$ Given the review's focus on identifying features that appear important to delivering and sustaining eHealth interventions at scale, initial matrices focused on summarizing the selected published papers in terms of how interventions were utilized, scaled, and sustained; perceived successes as described by the authors; and the study's challenges and limitations. Based on this analysis, six key dimensions emerged that were used to organize further analysis: population needs and priorities; an enabling environment in the form of local governance, infrastructure and policies; systems integration; partnership; implementation and implementers; and, finally, users. All six dimensions are influenced by catalysts, eg, external push or promotion factors that lie outside the local health system. Subsequent matrices explored specific features associated with each of these dimensions. Certain studies contributed insight and experience on each dimension, while others focused on one dimension in particular (ie, user's experience). Separate review matrices were developed for intervention studies and reviews using Microsoft Excel 2013.

\section{Results and discussion}

The search yielded a total of 690 articles and reviews, from which 28 peer-reviewed articles were identified for full text access after applying inclusion and exclusion criteria. Of these, eight intervention studies and six review papers were selected for final review. No randomized or nonrandomized trials met the inclusion criteria set by this review, which required that an eHealth intervention had been implemented at the district or provincial level and lasted for more than 1 year in duration. Among the eight articles selected, there were four case studies, two cross-sectional surveys, and two mixed method studies (Figure 1). Only one paper focused on eHealth interventions in Asia, and the rest on LMICs in Africa. Among the six review papers, three reported on interventions in Africa, one in Bangladesh, while the rest had a global approach.

Initial matrices used to summarize and compare papers are presented in Web Annex 2 and 3. Emerging from this descriptive assessment were six major domains around which subsequent analysis was focused to uncover key features that appeared important to achieving scale, sustainability and health improvement. It should be noted, however, that the extent to which these features actually impact the 


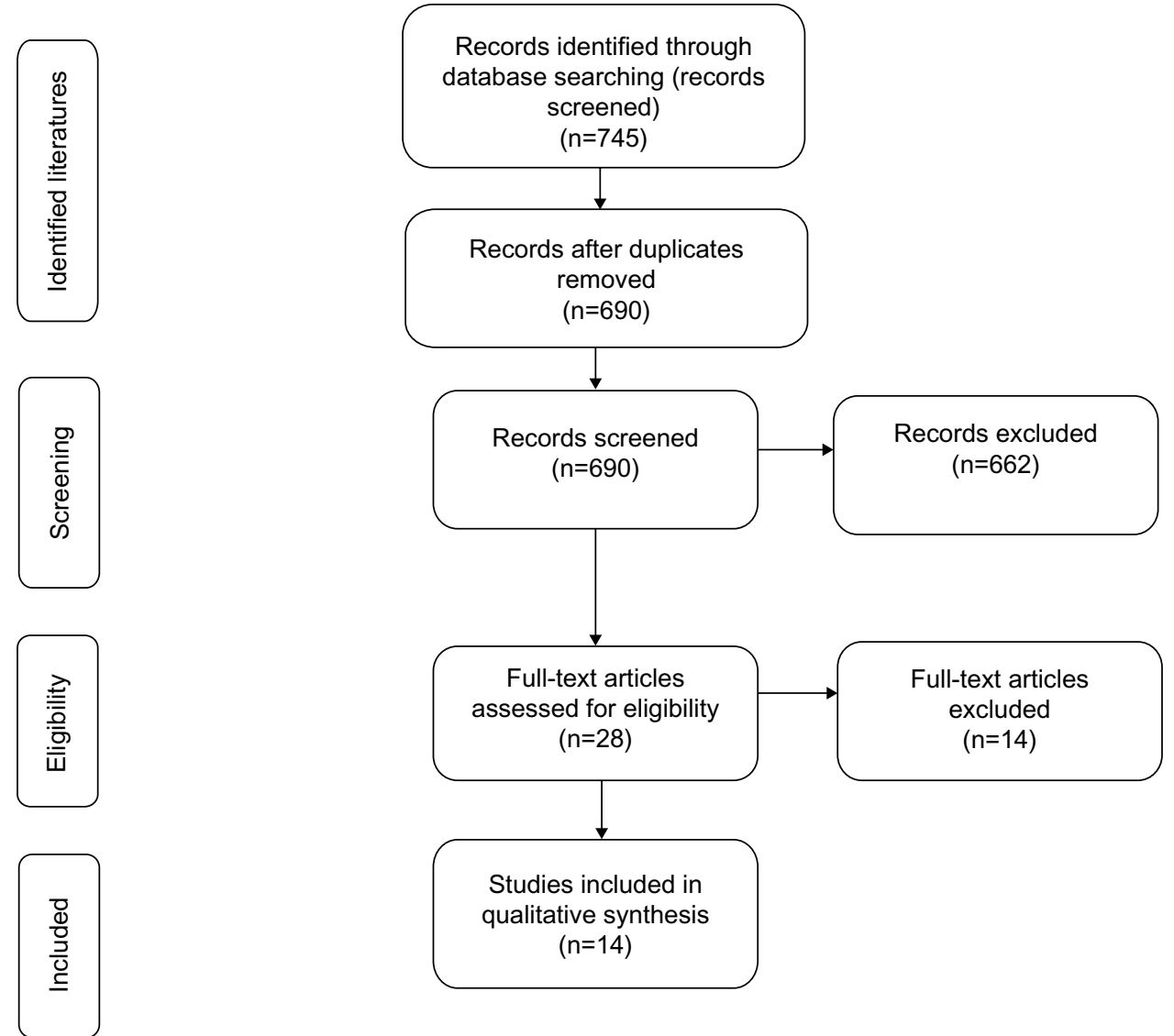

Figure I A prisma flowchart describing the search and inclusion process.

effectiveness of eHealth interventions in LMICs cannot be determined in the absence of rigorous evaluations, of which there are very few in the published literature.

Figure 2 displays six domains emerging from initial analysis of interventions, their relationships and constituent features of each. The model speculates that successful and sustainable eHealth interventions at scale are more likely to occur when they respond to real needs and priorities, where money and ideas are in place to innovate or catalyze a response to these needs, where an enabling environment is present, and when implementers and users are ready, willing and able to adopt and derive benefit from the innovation. We argue that these domains or dimensions are interlinked with one another and, in many cases, determine success in other dimensions. For example, commitment to addressing population needs and priorities cannot guarantee the success of an innovation. Rather, successful implementation of an eHealth intervention responds to population needs and requires catalysts in the form of innovative ideas, money, and infrastructure. The glue that holds all of these dimensions together is partnership, which ensures that eHealth innovations are shared investments, and systems integration, whereby innovations are embedded in institutions and processes, enabling scale and sustainability.

The following discussion reports on each of these dimensions, identifying key features that emerged from the eight papers selected for review. No one eHealth intervention reported on every dimension but, analyzed together, a number of shared features became apparent that appeared important to utilization, scale and sustainability. Findings from the six review papers provide supplementary evidence, where appropriate.

\section{Needs and priorities}

An initial dimension that emerged as important in many of the eHealth innovations considered in this review, and was integral to scale and sustainability, was the extent to which an innovation was responding to population needs and priorities (Figure 2). The importance of applying a needs-based approach when introducing new technologies into health systems is not new. ${ }^{19}$ Developing eHealth interventions in response to assessed needs and priorities helps ensure that an 


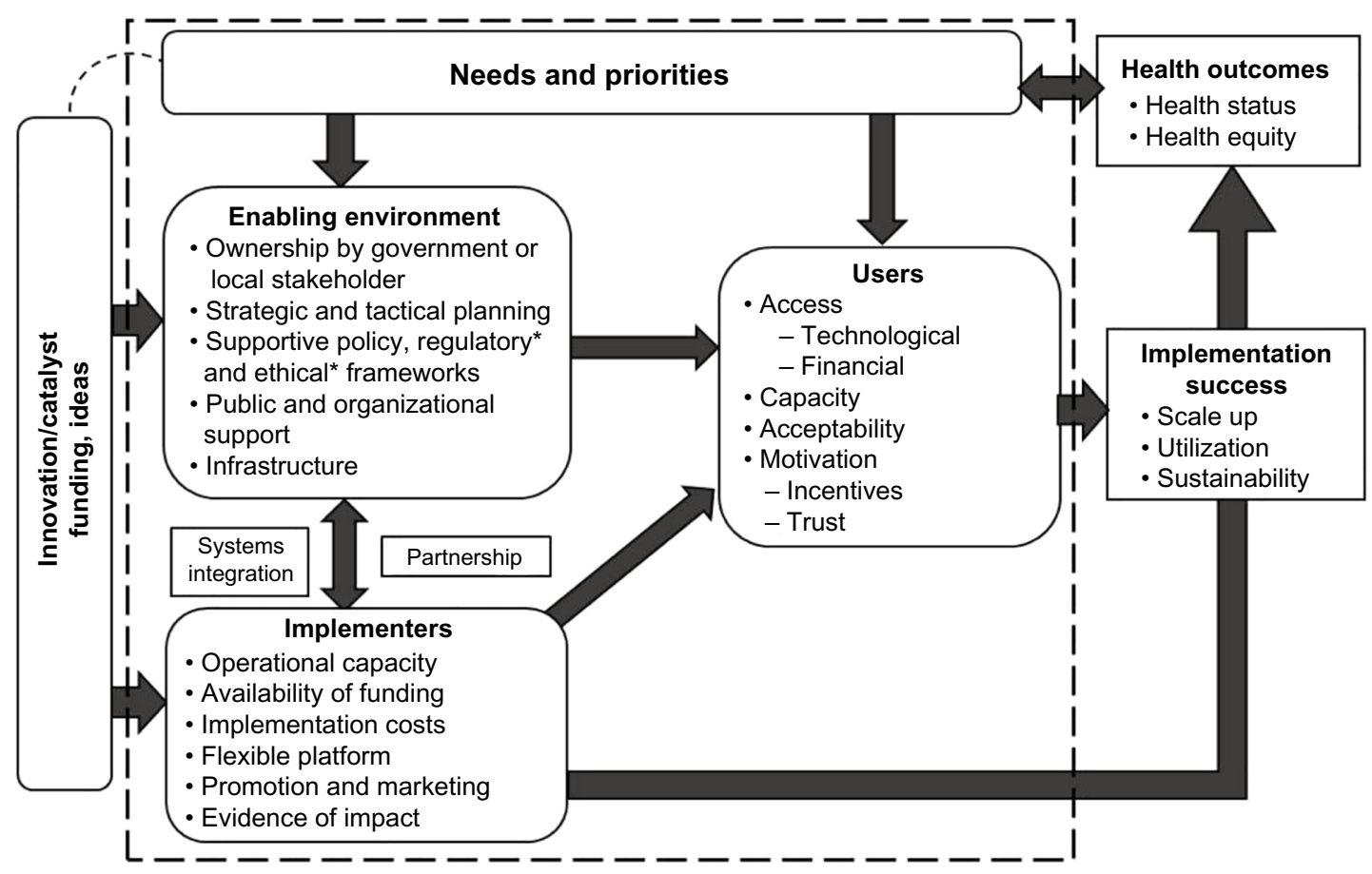

Figure 2 Features of eHealth innovations supportive of utilization, scale-up, and sustainability.

Notes: The identified domains are shown within the dashed square. The features marked with "*”" are deemed important but were not identified in the review.

intervention serves a purpose at a population level, that it is usable, scalable and sustainable for the intended population, and has the potential to satisfy a wider purpose in other population groups and contexts. For this reason, the first dimension identified in analysis included the specific population-level needs and priorities that each eHealth innovation intended to address. While it is difficult to ascertain the extent to which each intervention was successful in addressing population needs and priorities without supporting evaluations, any mention in papers of identified needs being addressed was considered in the review.

Interestingly, the focus of most of the large scale eHealth innovations in the review was oriented toward addressing priorities around improving health service coverage, and the reporting and use of health information. Telemedicine and other eHealth initiatives were used to compensate for health workforce shortages in remote areas where health services were restricted due to a lack of qualified and specialist health personnel (who were unable or unwilling to work in hard to reach areas) and where there was a lack of clinical infrastructure, especially for specialist care. ${ }^{20}$ Telemedicine was also used to provide local physicians with access to clinical information for medical decision making at the point of care and to support referral systems, facilitating the management of more complex cases. ${ }^{21,22}$ One of these projects, in north- ern India, found that telemedicine increased utilization of pediatric services because users recognized they could save money and time through the telemedicine system. ${ }^{22}$ Another example, the Virtual Doctor Project in Zambia, combined mobile health vans with the provision of specialist care at a distance. ${ }^{20}$ Each of the Zambian and Indian cases saw their projects as both an extension and augmentation of the existing primary health care system.

Also addressing supply side challenges, the Réseau en Afrique Francophone pour la Télémédecine (RAFT) Project developed a medical education and telemedicine network in 17 African countries to overcome the isolation of local health care providers working in remote areas and their limited opportunities for continuing medical education. ${ }^{23}$ The need to improve the quality, reporting and utilization of health information among health staff was the target of the health management information system (HMIS) component of a health systems strengthening project in Zanzibar, Tanzania. ${ }^{24}$ The authors found that improving data quality also improved the use of data in decision making.

A number of papers highlighted the importance of designing interventions based on government priorities and national health strategies. Without close alignment between these two areas, the coordination of intra-national initiatives within and between the public and the private sectors was 
seen as very difficult, and thereby impacted the potential for scalability. This priority was seen to influence the extent to which government and other stakeholders could be engaged during implementation, another key factor for success of an intervention.

\section{Enabling environment}

An enabling environment sensitive to the needs and priorities discussed in the previous section is important in creating the right conditions for eHealth efforts to be successful. An enabling environment has been described, broadly, as "a set of interrelated conditions - such as legal, bureaucratic, fiscal, informational, political, and cultural - that impact on the capacity of ... development actors to engage in development processes in a sustained and effective manner". ${ }^{25}$ While government is the main actor in creating the right conditions, given their key role in mandating, facilitating, resourcing and partnering in the implementation of eHealth projects, ${ }^{26}$ they are not the only ones with a role to play. The NGO, private and civil society sectors are also influential. Different factors that make up the enabling environment are likely to impact different health actors and implementers in different ways, with many interrelationships between them.

Ownership by local government and stakeholders emerged as a prime determining factor influencing the outcome and success of the eHealth innovations examined in this review. ${ }^{20,21,24,27}$ A project can be owned by government, local and external stakeholders, as well as through partnership or consortia arrangements. However, the greater the extent of government and local ownership or involvement, the more likely it appears that an innovation will achieve scale and sustainability. In the Botswana telemedicine project, the Ministry of Health was the principal stakeholder, contributing $84 \%$ of the operational budget for the first year, but customization and ownership by local stakeholders was also important. This project was scaled up gradually from eleven to 25 locations, including districts and tertiary hospitals. Similar experiences regarding the importance of ownership were also suggested in the review by ArandaJan et al. ${ }^{28}$ Without government ownership and oversight of eHealth innovations, including their inclusion into a comprehensive health strategy, eHealth projects run the risk of being implemented in ad hoc ways by nongovernment actors, typically through funding sources that tend not to be sustained beyond the project lifecycle.

Strategic and tactical planning during development and implementation was another key factor identified for the success of eHealth innovations. This refers to the practical advance planning that is needed to ensure that adequate infrastructure, resources and staff are available to operationalize an innovation, including planning for training, and managing the likely impact on human resources. ${ }^{29}$ The authors highlight that there are many factors that arise in LMICs which would not need to be considered in resource-rich countries. Appropriate design and timely decision making were reported as pivotal.

Public and organizational support of projects can play an important role in the progress of eHealth innovations. Mupela et al describe how the Zambian government's decision to buy nine mobile clinics from the People's Republic of China for a large sum of money raised controversy among the public who felt that rural health centers would have been a better investment of public funds. ${ }^{20}$ The decision to procure the mobile clinics was made as part of a high level aid deal, with no security for ongoing investment to sustain them, and without the involvement of public representatives or parallel educational initiatives to demonstrate how the mobile clinics could benefit rural people. This example raises questions about the government's commitment to health system strengthening. Clearly, political will, the decision-making process of implementers or policy makers, and the manner in which these are perceived by the public have an important impact on the success of an innovation, and should be taken into account in their planning, design, and implementation.

This need for public support of eHealth innovations also applies at the organizational level to health employees, whether these are clinical staff or administrators. Acceptance can be difficult in organizations that have a strong hierarchical structure and traditions. Thus, Lluch ${ }^{14}$ suggests that health care organizations need to be aware of how existing organizational structures and staff will receive and cope with innovations and, if necessary, restructure themselves to create a more flexible workplace culture that is not only accepting of new technologies but able to fully leverage their potential.

Supportive and comprehensive health policy frameworks comprised of all the factors mentioned earlier (government ownership, strategic planning, gaining acceptance from public and professional users of eHealth innovations) were found to provide a strong foundation for successful implementation. A lack of standardized operational frameworks for eHealth initiatives across different government departments has been reported as a major barrier for eHealth innovations. ${ }^{13}$ Such a situation, all too typical, prevents coordination, potentially 
duplicates resources, and makes it difficult to assess how different innovations compare in terms of implementation effectiveness or outcomes.

\section{Infrastructure}

The studies included in this review covered a wide range of technological approaches used for the application of eHealth services. Access to technology and technological infrastructure in various forms is clearly a prerequisite for eHealth innovations, but coverage and reliability are major challenges. ${ }^{28}$ Half of the studies and all but one of the literature reviews included in this review were based on mobile phone applications, with projects taking advantage of widespread access to mobile phones. For example, ninety-five percent of people in Botswana are estimated to have access to a mobile phone. ${ }^{21}$ In Bangladesh, an mHealth study with data from 2012 showed that $81 \%$ of households had access to at least one mobile phone and $45 \%$ of the adult population owned their own mobile phone. ${ }^{30}$

While mobile phones are becoming ubiquitous across LMICs, it is important not to take their ownership for granted. The poorest in the population and women, in particular, have less access to mobile phones compared to higher socioeconomic groups and men. ${ }^{30}$ Intermittent network connectivity and mobility problems have also been reported. ${ }^{23}$ The Virtual Doctor Project in Zambia used satellite technology because network coverage was poor and unreliable in the target regions and telephones were not so widely used. ${ }^{20}$

Other than innovative technology, eHealth innovations cannot bypass the need for the type of infrastructure that is taken for granted in high income countries, including road and transportation networks and electricity. ${ }^{21,29}$ Roads can be rendered inaccessible from flooding and the impact of other climate issues, as reported by the Virtual Doctor Project in Zambia. ${ }^{20}$

\section{Systems integration}

Systems integration is a feature of many of the large-scale eHealth innovations reviewed here. Several degrees of integration come into play under this feature. In the case of OpenMRS programs, or programs that are adjunct to existing clinical practice, it is important to effectively integrate the innovation into the daily work flow of clinicians ${ }^{29}$ or other user groups. Likewise, several papers in the review emphasized the importance of ensuring that new technologies be interoperable with existing systems, especially where parallel IT systems projects are underway. An eHealth intervention will have little chance of scaling-up if the technologies it employs cannot be linked to systems already in place or being planned at a national or regional level. The example of the Millennium Village Project (MVP), where different health information systems were being implemented in different sites, highlights both the technical challenge and scale of this problem. ${ }^{27}$

A broader level of integration involves the alignment of eHealth interventions with national health system strategies and their integration into existing government health programs. ${ }^{21,23}$ This kind of integration often depends on strong organizational and personal networks, concerted efforts to create stakeholder ownership, and the securement of government support. Stakeholder consultation in the conception and design of indicators in alliance with the existing HMIS is one approach toward system integration evident in the Zambian telemedicine project. ${ }^{20}$ Experience from Botswana illustrates how long-term government support and funding for systems integration is important to sustainability. ${ }^{21}$

\section{Partnership}

Evidence from our review suggests that effective partnership between public, private and nonprofit sectors is a distinguishing feature of successful eHealth interventions, helping to fund and catalyze the process of development, implementation, or expansion of eHealth projects. ${ }^{21,24,28}$ In Botswana, a major financial investment by the Government in a telemedicine intervention to expand specialist care coverage was implemented through a partnership with the University of Pennsylvania, the Botswana-University of Pennsylvania Partnership (BUP). The project also involved a key partner from the private sector, Orange, one of the largest mobile networks in the world, which provided logistical and technical support. This intervention is an example of an eHealth innovation that is in the process of being scaled up in other parts of the country, and shows promise in improving access to care, empowering providers, and reducing referral and associated costs. ${ }^{21}$ In a different example from Zanzibar, Tanzania, district health teams from primary care units, hospitals, and other health programs across the island were brought together for data use workshops to strengthen and consolidate the national HMIS. ${ }^{24}$ During a series of workshops, health staff from different districts assessed and critiqued their own and colleagues' health data. The workshops improved collaboration between different district health teams and led to the integration of a unified district health information system (DHIS), including a single set of national health indicators. The workshops also allowed participants to better understand the roles of different health colleagues from their own 
and other districts. Interestingly, the Zanzibar project also provided lessons about the difficulty of developing projects being funded by different donors. They reported how early integration was slow because some health programs within districts were funded by external donors and this resulted in these teams being initially unwilling to share what they considered to be "their" data.

Many of the eHealth studies identified challenges related to operating costs, infrastructure, technical competency and policy support that necessitated external partnerships for development, implementation, advocacy and expansion. Experience also suggested that it is important to consider the role and implications of partnerships prior to engagement. One example of this is whether dependence on donor partners for funding or external technological support will limit long-term sustainability. ${ }^{21,28}$ In Botswana, agreements between the government and the private telecommunications group, Orange, were contingent on mutual satisfaction with performance, emphasizing the complexity of managing such relationships. The mHealth project in Botswana was also compromised by the departure of key contributors and supporters, and high staff turnover. In several instances, the sudden transfer of employees without proper delegation of responsibilities created management and communication problems among key stakeholders. ${ }^{21}$ In short, while external partnerships may be important, it appears that eHealth innovations are most likely to be sustained in the context of local ownership and government support. ${ }^{21,24,28}$

\section{Implementation requirements}

The management and operational capacities of those implementing eHealth interventions are among the most crucial drivers of success. Six features were identified as vital for effective implementation of the eHealth interventions reviewed in this paper.

Operational capacity, including a competent technical team backed by necessary logistics, financing and equipment, are critical requirements to ensure that all aspects of project delivery are in place for a successful eHealth program. Securing the right mix of trained personnel who have the ability to effectively implement an intervention and address administrative and technical issues is vital but often challenging. ${ }^{29}$ The expertise required for staffing eHealth programs in LMICs differs from regular health programs because they require both medical personnel as well as IT programmers and support staff. The arrangement of staff is, however, a function of organizational need. For instance, RAFT employs local level teams in each participating country coordinated via a central team based in Switzerland. The local team is comprised of a national focal point who supervises all operations and liaises with the government; a medical coordinator who trains and supports local health care providers and assesses local educational needs and opportunities; and a technical coordinator/programmer to oversee routine functioning of the telemedicine tools. ${ }^{23}$

Capacity to manage and troubleshoot is also essential. This requires the input of software programmers with responsibility for designing, implementing and maintaining the technical platform, as well as working with users to resolve technical issues such as software bugs, device malfunction, and hacking attempts. Dependence on individual capacity and/or out-of-country experts may run the risk of system disintegration in the event that a programmer leaves; hence the importance of building in-country capacity around ICT development and support for long-term sustainability. For example, the Botswana team sourced its development, maintenance and support from local IT developers who used open source technologies. ${ }^{21}$ Another solution proposed by Kanter was to share platform development with partnering organizations to reduce risk of dependence. ${ }^{27}$

Availability of funding is one of the most important determinants of innovation success. Lack of funding or inappropriate allocation of available financial resources can derail even the most promising innovations. ${ }^{28}$ The source of financial support is also key to sustainability. For example, the Botswana telemedicine service was principally funded by the Ministry of Health, and a cost-sharing plan between local partners, government, and other donors. Coverage is gradually being increased to include specialist services. ${ }^{21}$ By contrast, the Virtual Doctor Project in Zambia relied on charity-based donations, posing a risk to long-term sustainability. ${ }^{20}$ Persistent reliance on donor support was also reported as a challenge by Lewis et al in their review paper. ${ }^{31}$ Despite this threat, $47 \%$ of eHealth interventions in LMICs are donor dependent, emphasizing the need to promote alternative sources of funding, such as government contracts, insurance, or direct payments from consumers, for scalability. ${ }^{31}$ To secure more sustainable sources of funding such as government support, eHealth interventions need to gather credible information on cost and effectiveness to justify why they warrant state investment. ${ }^{20,21} \mathrm{~A}$ sense of local ownership is also paramount to state buy-in and arguments for the incorporation of eHealth into the national budget. ${ }^{21}$

Implementation costs, which take into account all human resources and operational costs for designing eHealth 
platforms, purchasing equipment, and maintenance, must be affordable for eHealth scale-up to occur. eHealth programs, such as the MVP, and patient record systems in countries such as Kenya, Rwanda, and South Africa, have managed to curtail costs by using open source platforms such as Free/Libre Open Source Software for Health Care (FLOSS-HC) and OpenMRS. ${ }^{27,29}$ MVP also reports collaborating with partners to reuse codes and develop eHealth platforms that save time and money. ${ }^{27}$ Cost savings with respect to human resources also make eHealth innovations easier to justify and scale up. According to Mupela et al, the annual cost of each virtual doctor system in Zambia was approximately half the cost of an average doctor's salary. ${ }^{20}$ However, maintenance costs and finances for on-the-ground support need to be considered even after cost savings, and are often associated with external enabling factors such as infrastructure and internet connectivity in the country. ${ }^{27,29}$ It should also be noted that delegating costs to users of eHealth innovations may jeopardize sustainability ${ }^{28}$ while cost savings to patients, both in terms of time and money, may encourage an innovation's continued uptake.

A flexible and interoperable health platform is another factor enabling implementation scale-up. ${ }^{27}$ The MVP employed an open source platform, FLOSS-HC, because of its lower cost and greater potential for customization compared to proprietary products. ${ }^{27}$ However, open source platforms generally need higher levels of developer support. Although FLOSS applications used by MVP were backed by a volunteer developer community, they opted to build a support team for more consistent assistance.

As eHealth programs become common within and across countries and continents, there is practical value in ensuring interoperability across different platforms to maintain information flow and contribute to HMIS. The MVP used a centralized concept dictionary for data element mapping that allowed semantic congruence across the different languages used in its 31 sites in Sub-Saharan Africa. ${ }^{27}$ In India, the telemedicine center at PGIMER, Chandigarh, supported the development of telemedicine technology that has been adopted by at least three hospitals, and has informed other telemedicine systems in the country. ${ }^{22}$

Other managerial skills to address nontechnical issues, both internal and external, are important within the team. The team should be able to apprehend and solve problems, such as solutions to power shortages, poor internet capabilities, hardware breakdown, security breaches, strikes, staff turnover, etc. The implementers of OpenMRS in some of the seven African countries participating in the MVP had to utilize solar panels and generators to overcome electricity issues. When faced with a security breach, the BUP set the standards for data encryption using the USA's Health Insurance Portability and Accountability Act. ${ }^{21}$ Management difficulties of this nature are often commensurate with country-level infrastructural shortcomings and lack of national IT standards, emphasizing the important role of the enabling environment (Figure 2).

Innovation promotion and marketing also appears important to innovation uptake. Unfamiliarity and skepticism about eHealth products can be initial hurdles to uptake and sustainability. The RAFT network overcame these barriers through a continuing education program. ${ }^{23}$ The BUP mHealth projects in Botswana assigned "specialty managers" to raise awareness on telemedicine and eHealth among health professionals through workshops and sensitization programs. Mupela et al suggest that the benefit of an innovation/intervention should be publicized in order to facilitate utilization and integration into mainstream health care delivery systems, and to encourage user uptake. ${ }^{20}$

Evidence on impact and cost-effectiveness is imperative to justify investments in eHealth programs. Kanter et al recommend incorporation of cost-benefit analysis into monitoring and evaluation frameworks for eHealth projects. ${ }^{27}$ However, only one of the studies considered in this review explicitly cited cost savings as a result of an eHealth intervention in the area of telemedicine. ${ }^{22}$ In fact, the lack of empirical evidence has been described as a roadblock to the scaling-up of eHealth programs in reviews by Aranda-Jan et al and Bloomfield et al. ${ }^{12,28}$ eHealth programs can only be considered essential for health systems in the presence of credible evidence on cost, performance and health outcomes. ${ }^{10}$

\section{Users}

In this review, we identified two groups of users of eHealth products. Demand side users, those benefiting from services, such as patients; and supply side users, including the health care providers who employ the innovation or technology in the delivery of services. The affordability of and access to the technological tools used in eHealth projects, such as mobile phones, are known issues that need to be taken into account in innovation design. Other factors important to understanding intervention uptake and sustainability include factors related to the users' ability to access and utilize the eHealth service or technology and its acceptability (issues of motivation, trust). In addition to these factors, recent eHealth reviews also identified issues of privacy and confidentiality as potentially 
important influences on the acceptability and use of eHealth interventions.

The capacity of users is an important determinant of whether an eHealth technology is successfully utilized. For example, providers' technical ability to employ OpenMRS software was reported as a successful implementation factor. ${ }^{29}$ Technical training and demonstrating the benefits of the innovation are essential, ${ }^{23}$ as are efforts to make the technology as culturally appropriate as possible by means of local customization in terms of language and appearance. ${ }^{21}$ If a user is not comfortable with the technology itself, the acceptability of the technology is compromised. For example, Bagayoko et al reported that the high degree of comfort with and acceptance of mobile phones as a communication medium was a factor underlying the perceived success of the RAFT project. $^{23}$

For supply side users such as health providers, educational background, training and work experience were perceived as particularly important. ${ }^{29}$ Review papers also emphasized the critical role of individual capacity, team coordination, and availability of technical and administrative support to the successful implementation of eHealth innovations. ${ }^{12-14}$

The acceptability and adoption of eHealth interventions can also be increased through early engagement of users in intervention design and the use of feedback mechanisms. In Zanzibar, Tanzania, utilization of the HMIS at the district health management level was enabled by a participatory workshop whereby representatives from different districts or programs presented their respective performance data on a regular basis. This approach created a competitive but constructive opportunity to review progress, provide feedback, and enhance DHIS data quality and utilization. ${ }^{24}$ A stepped approach to the implementation of eHealth interventions was also reported to increase acceptability, allowing users time to adapt and gain proficiency. ${ }^{27}$

Motivating potential users with incentives is another approach to encourage the uptake and adoption of eHealth innovations. In the RAFT project, health care providers requested formal recognition for participating in a distance education program. In response, project implementers have been developing a module using a virtual Internet Patient Simulator that allows operational knowledge to be assessed and converted into formal credentials. ${ }^{23}$ From the patient perspective, a recent customer survey from a long-running telemedicine initiative in Bangladesh, revealed the importance of responsive, empathetic and courteous service in motivating continued uptake. ${ }^{32}$ Irrespective of user type, a lack of motivation or incentives has been reported to negatively influence the implementation outcomes of eHealth innovations. ${ }^{14,31}$

Trust has been identified in a recent review of eHealth interventions as critical in determining the end user's experience and their willingness to take up an innovation. ${ }^{14}$ To maintain trust, transparency is needed so that all concerned understand the implications of the technology for their roles and status. For example, concerns that eHealth would replace onsite doctors, or end user perceptions that the intervention was a response to lack of onsite staff, were misunderstandings that created implementation challenges in the telemedicine component of the RAFT project. ${ }^{23}$ In such cases, the involvement of colleagues and peers in motivating new users was reported to have a positive effect. ${ }^{21}$

Another factor influencing trust in eHealth technologies is the security and confidentiality of patient data. In their review paper, Aranda-Jan et al suggest that telemedicine provides an effective platform for sharing sensitive information in contexts where face-to-face information sharing may lead to stigma. ${ }^{28}$ However, ensuring that patient information is secure, and their right to privacy and confidentiality is honored by health care providers, are important considerations in systems design.

\section{Limitations}

One of the principal limitations of this review was the lack of published evidence on the impact of eHealth interventions in LMICs. There were few randomized and nonrandomized control trials available to review and very few published studies that evaluated impact. Among the handful of trials or evaluations identified, none met our inclusion criteria, given our specific focus on large scale innovations and peerreviewed articles. In the absence of strong evidence, our analysis was confined to describing the probable linkages between the key dimensions identified in the review, and their likely impact on eHealth implementation, use, scale-up, and sustainability. We acknowledge that experience from smaller scale interventions might also provide valuable lessons. Limiting our review to peer-reviewed literature meant that we did not consider the gray literature and reports about eHealth projects that might have added more dimensions to the article and provided a wider variety of examples of eHealth projects which have been, or are in the process of being, implemented.

\section{Conclusion}

eHealth has been increasingly advocated as a promising area of innovation to address health system challenges but 
there have been few reviews of large-scale projects assessing the factors affecting eHealth utilization, scale-up, and sustainability. This literature review of large-scale eHealth innovations in LMICs identified six key dimensions needing careful consideration in the planning, implementation, utilization, and scale-up of eHealth projects: that eHealth projects need to be designed in response to identified health needs and priorities; be supported by an enabling environment; ensure IT systems integration; establish effective partnership between stakeholders; ensure implementation requirements are met; and pre-empt and address issues related to end users' abilities to access, trust, accept, and utilize an eHealth product. In contrast to existing reviews, these dimensions provide a system-wide perspective on the requirements needed for taking eHealth projects forward to achieve adoption, scale, and sustainability.

There is a need for further research to provide trusted data on cost-effectiveness, efficacy and impact of eHealth projects, ${ }^{33,21}$ as well as greater clarity on the external validity and study design of projects. It is observed that over $70 \%$ of African countries have implemented at least one eHealth initiative but less than $10 \%$ of these countries have evaluated these initiatives. When reported, most of the studies only present descriptive outcomes limiting the scope of evidence to justify scale-up or inform decision making. ${ }^{21,24,28}$ This gap is hopefully one which will be addressed as the field of eHealth grows and is further established.

There were several issues not addressed in this literature review but which may be important to consider. One is the need for regulatory frameworks for eHealth that protect patients (or data subjects) from the misuse and mismanagement of personal data, and ensure that there are clear limitations on data collection, storage and use. Ethical frameworks are a key part of regulation but not just for maintaining confidentiality. When patients are recruited to use eHealth products, there needs to be genuine informed ethical consent. This means ensuring that eHealth users fully understand the implications of participation and what will happen to the personal data they provide before they participate. In the context of many LMICs, there is a danger that existing systems of health governance are too weak to control and enforce any regulatory frameworks if eHealth projects take off. The security of personal data is of particular concern in relation to the ongoing growth of other sectors, such as finance and insurance, given the risk of data (mis) use in the development of these business markets.

Another dimension not found in the literature reviewed but known to be important ${ }^{34}$ is how gender inequality and social and cultural norms, especially gender power relations and bargaining at the household level, affect women's ability to access and use mobile phones, and their perceptions toward mobile phone use for health purposes. Mobile phone access or ownership is not just a matter of affordability, technical literacy, or network coverage, but is also affected by complex social and cultural factors at the household and community level, including gender. These issues need to be thoroughly understood well in advance of project implementation, and measures for addressing them incorporated into project designs.

LMICs are in a strong position to develop eHealth strategies because eHealth is still in its infancy in these countries. Progress requires curbing the current trend of "pilotitis", and adopting a more strategic approach to thinking about the possibilities of eHealth innovations in relation to national and regional health needs, a point also made by Ahmed et al. ${ }^{13}$

\section{Disclosure}

The authors report no conflicts of interest in this work.

\section{References}

1. World Health Organization. Global Health Workforce Shortage to Reach 12.9 million in Coming Decades. Geneva: World Health Organization; 2013. Available from http://www.who.int/mediacentre/news/ releases/2013/health-workforce-shortage/en/. Accessed December 14, 2014.

2. United Nations foundation $\&$ Vodafone Foundation. mHealth for development: the opportunity of mobile technology for healthcare in developing world. United Nations Foundation, Washington, DC. and The Vodafone Foundation, Newbury, UK; 2009. http://www.vitalwaveconsulting.com/ pdf/2011/mHealth.pdf. Accessed December 15, 2014.

3. Franz-Vasdeki J, Pratt BA, Newsome M, Germann S. Taking mHealth solutions to scale: enabling environments and successful implementation. J Mob Technol Med. 2015;4(1):35-38.

4. Simmons R, Fajans P, Ghiron L. Scaling up Health Service Delivery: from Pilot Innovations to Policies and Programmes. Geneva: World Health Organization; 2007.

5. World Health Organization. E-Health. Available from http://www.who. int/trade/glossary/story021/en/. Accessed February 11, 2015.

6. Kay M. mHealth: New Horizons for Health through Mobile Technologies. Geneva: World Health Organization; 2011:6.

7. International Telecommunication Union. The World in 2014: ICT facts and figures. International Telecommunication Union, Geneva; 2014; http://www.itu.int/en/ITU-D/Statistics/Documents/facts/ ICTFactsFigures2014-e.pdf. Accessed March 1, 2015.

8. Curioso WH, Kurth AE. Access, use and perceptions regarding Internet, cell phones and PDAs as a means for health promotion for people living with HIV in Peru. BMC Med Inform Decis Mak. 2007;7:24.

9. Kahn JG, Yang JS, Kahn JS. 'Mobile' health needs and opportunities in developing countries. Health Aff (Millwood). 2010;29(2): 252-258.

10. Labrique AB, Vasudevan L, Kochi E, Fabricant R, Mehl G. mHealth innovations as health system strengthening tools: 12 common applications and a visual framework. Global Health Sci Pract 2013;1(2):160-171.

11. Chang LW, Kagaayi J, Arem H, et al. Impact of a mHealth intervention for peer health workers on AIDS care in rural Uganda: a mixed methods evaluation of a cluster-randomized trial. AIDS Behav. 2011;15(8): $1776-1784$. 
12. Bloomfield GS, Vedanthan R, Vasudevan L, Kithei A, Were M, Velazquez EJ. Mobile health for non-communicable diseases in SubSaharan Africa: a systematic review of the literature and strategic framework for research. Global Health. 2014;10:49.

13. Ahmed T, Lucas H, Khan AS, Islam R, Bhuiya A, Iqbal M. eHealth and mHealth initiatives in Bangladesh: a scoping study. BMC Health Serv Res. 2014;14:260.

14. Lluch M. Healthcare professionals' organisational barriers to health information technologies-a literature review. Int J Med Inform. 2011;80(12):849-862.

15. Elbert NJ, van Os-Medendorp H, van Renselaar W, et al. Effectiveness and cost-effectiveness of eHealth interventions in somatic diseases: a systematic review of systematic reviews and meta-analyses. $J \mathrm{Med}$ Internet Res. 2014;16(4):e110. http://www.jmir.org/2014/4/e110/. Accessed February 12, 2015.

16. Mariani AW, Pêgo-Fernandes PM. Telemedicine: a technological revolution. Sao Paulo Med J. 2012;130:277-278.

17. Kay M, World Health Organization. Building Foundations for EHealth: Progress of Member States. Report of the WHO Global Observatory for EHealth. Geneva: WHO Press; 2006.

18. Gale NK, Heath G, Cameron E, Rashid S, Redwood S. Using the framework method for the analysis of qualitative data in multi-disciplinary health research. BMC Med Res Methodol. 2013;13:117.

19. World Health Organization, Pan American Health Organization, University of Ottawa, Canadian Coordinating Office for Health Technology Assessment. Needs based tool kit for health technology assessment. Paper presented at Needs-Based Technology Assessment: Exploring Global Interfaces 1994; Ottawa. Available from: http://www.cgh. uottawa.ca/whocc/projects/nb_toolkit/nb_toolkit.pdf. Accessed March $1,2015$.

20. Mupela E, Mustard P, Jones H. Telemedicine and Primary Health: The Virtual Doctor Project Zambia. UNU-MERIT, Maastricht Economic and Social Research and Training Centre on Innovation and Technology; 2011.

21. Littman-Quinn R, Mibenge C, Antwi C, Chandra A, Kovarik CL. Implementation of $\mathrm{m}$-health applications in Botswana: telemedicine and education on mobile devices in a low resource setting. J Telemed Telecare. 2013;19(2):120-125.

22. Singh M, Das RR. Four years of experience of telemedicine for paediatric care in three Punjab hospitals, North India: achievements and lessons. Postgrad Med J. 2010;86(1022):688-691.

23. Bagayoko CO, Perrin C, Gagnon MP, Geissbuhler A. Continuing distance education: a capacity-building tool for the de-isolation of care professionals and researchers. J Gen Intern Med. 2013;28 Suppl 3: S666-S670.
24. Braa J, Heywood A, Sahay S. Improving quality and use of data through data-use workshops: Zanzibar, United Republic of Tanzania. Bull World Health Organ. 2012;90(5):379-384.

25. Thindwa J. Enabling environment for Civil Society in CDD Projects", Washington, DC: World Bank, Social Development Family, CDD Learning Module, 2001. Available at: http://www.worldbank.org/participation/ enablingenvironment/EnablingenvironmentCECDD.pdf.

26. Brinkerhoff DW. The enabling environment for implementing the millennium development goals: government actions to support NGOs. Paper presented at the George Washington University Conference "The Role of NGOs in Implementing the Millennium Development Goals", Washington, DC, May 12-13, 2004.

27. Kanter AS, Borland R, Barasa M, et al. The importance of using open source technologies and common standards for interoperability within eHealth: perspectives from the Millennium Villages Project. Adv Health Care Manag. 2012;12:189-204.

28. Aranda-Jan CB, Mohutsiwa-Dibe N, Loukanova S. Systematic review on what works, what does not work and why of implementation of mobile health (mHealth) projects in Africa. BMC Public Health. 2014; $14: 188$.

29. Mohammed-Rajput NA, Smith DC, Mamlin B, Biondich P, Doebbeling BN. OpenMRS, A Global Medical Records System Collaborative: factors influencing successful implementation. AMIA Annu Symp Proc. 2011;2011:960-968.

30. Khatun F, Hanifi S, Iqbal M, et al. Prospects of mHealth services in bangladesh: recent evidence from Chakaria. PloS one. 2014;9(11): e111413.

31. Lewis T, Synowiec C, Lagomarsino G, Schweitzer J. E-health in low- and middle-income countries: findings from the Center for Health Market Innovations. Bull World Health Organ. 2012;90(5):332-340.

32. Akter S, D'Ambra J, Ray P. Service quality of mHealth platforms: development and validation of a hierarchical model using PLS. Electronic Markets. 2010;20(3-4):209-227.

33. Wootton R, Geissbuhler A, Jethwani K, et al. Comparative performance of seven long-running telemedicine networks delivering humanitarian services. J Telemed Telecare. 2012;18(6):305-311.

34. GSMA mWomen Programme. Striving and surviving: exploring the lives of women at the base of the pyramid. London: GSMA mWomen; 2012 .
Innovation and Entrepreneurship in Health

\section{Publish your work in this journal}

Innovation and Entrepreneurship in Health is an international, peer reviewed, open access journal publishing original research, reports, reviews and commentaries on innovation and entrepreneurship in health. Special focus will be given to the theory, process, and practice of innovation and entrepreneurship by individuals and organizations

\section{Dovepress}

within the health care context globally. The manuscript management system is completely online and includes a very quick and fair peer review system, which is all easy to use. Visit http://www.dovepress.com/ testimonials.php to read real quotes from published authors. 